\title{
Effective ventilation strategies for acute respiratory distress syndrome
}

\author{
Thomas E Stewart MD FRCPC
}

$\mathrm{I}^{\mathrm{n}}$ n 1993, a consensus conference on mechanical ventilation concluded that mechanical ventilation for patients with acute respiratory distress syndrome (ARDS) using tidal volumes that generate high airway pressures (in particular, plateau pressures $35 \mathrm{~cm} \mathrm{H}_{2} \mathrm{O}$ or greater) is concerning (1). They went on to recommend that tidal volumes should be reduced from classic levels to as low as $5 \mathrm{~mL} / \mathrm{kg}$ (possibly in conjunction with permissive hypercapnia) if necessary to avoid high airway pressures. These recommendations were made based on animal and emerging uncontrolled human data suggesting that ventilator-induced lung injury was a serious concern that could affect patient outcome. It should be noted that these recommendations were made with the provision that there was a need for conclusive evidence. Since then, the evidence in regard to ventilator-induced lung injury has evolved, so that today, not only are low tidal volumes recommended, but efforts to prevent cyclic collapse and reopening of lung units, as well as strategies to open the lung, are suggested (2).

To date, five randomized, controlled trials have been published (more have been concluded or are underway, including the Lung Open Ventilation Strategy [LOVS] being conducted in 25 Canadian centres) evaluating the use of lung protective ventilation strategies in patients with ARDS or acute lung injury, and/or at high risk for ARDS (3-7). Two of the studies found a significant difference in mortality favouring the lung protective study arms $(3,4)$ and three did not $(5-7)$. However, one of the positive studies demonstrated a very high mortality rate in the control arm, and it has generally been accepted that this arm accounted for much of the mortality difference, despite the fact that the intervention arm looked reasonably strong (3). Given the fact that the control arm likely represented a common approach to mechanical ventilation, this was very important information, and the publication of this study (3) demonstrated that mechanical ventilation practice impacts patient outcome. The other study that found a difference in mortality was conducted by the the Acute Respiratory Distress Syndrome Network (ARDSnet) in the United States (4). This study was designed to simply evaluate the role of pressure and volume limitation in ARDS; however, given that the strategy included respiratory rates of up to 35 breaths/min to manage respiratory acidosis, it is interesting to note (by virtue of gas trapping) that it may have also achieved a lung open approach (8). The publication of these important trials should have led critical care teams to take inventory of their mechan- ical ventilation approaches and make necessary modifications to ensure optimal patient outcome. Because the ARDSnet study is the largest and perhaps most important to date, some may find it disappointing that acceptance of this protocol has been slow, even in centres involved in the original trial. However, there may be an alternate explanation.

Recently, a group of investigators reported a meta-analysis of the five trials on lung protective ventilation to date (9). They demonstrated that in the control arms of the two studies that found a significant mortality difference $(3,4)$, plateau pressures were much higher than in any of the other studies (5-7). Based on this, they suggested that the cumulative evidence shows that rather than adopting the protocol used in the ARDSnet intervention arm, clinicians should be focused on using moderate tidal volumes that avoid high plateau pressures (in the range of 30 to $35 \mathrm{~cm} \mathrm{H}_{2} \mathrm{O}$ ) (9). During mechanical ventilation, concern with plateau pressures in this range is certainly supported by recent data that found that plateau pressures in excess of $35 \mathrm{~cm}$ $\mathrm{H}_{2} \mathrm{O}$ are independently associated with mortality (10). Although I tend to agree that the culmination of data in this area supports the notion that clinicians should be more focused on avoiding high airway pressures than following the precise ARDSnet protocol, it should be pointed out that this approach has not yet been proven. Thus, the ARDSnet approach (4) is the standard against which all future lung protective strategies should be tested. Unfortunately, the authors of the above-mentioned meta-analysis (9) went a step further and suggested that because the control arms of the two trials that found a difference had higher tidal volumes than the investigators were using at the time of randomization, their importance was minimized and perhaps unethical. Such a suggestion has caused the suspension of important critical care research (11), with which I strongly disagree, as I have commented previously (12). It is important to reiterate that the initial consensus conference (although advocating airway pressure limitation) requested proof to this effect (1). If the randomized, controlled studies completed to date had not been performed (in particular, the ARDSnet study [4]), we would not have the necessary information to encourage change in ventilation practice, to the detriment of many patients. Clinicians should take inventory of the mechanical ventilation practice in their own institutions and ensure that appropriate modifications have been carried out. In addition, we need to encourage continued research in this area, because the mortality rate remains unacceptably high.

Critical Care, Mount Sinai Hospital, and University Health Network, University of Toronto, Toronto, Ontario

Correspondence: Dr TE Stewart, Mount Sinai Hospital, 600 University Avenue, Suite 1818, Toronto, Ontario M5G 1X5. Telephone 416-586-8774, fax 416-586-5981, e-mail tstewart@mtsinai.on.ca 


\section{REFERENCES}

1. Slutsky AS. Mechanical ventilation. American College of Chest Physicians' Consensus Conference. Chest 1993;104:1833-59.

2. Froese AB. High-frequency oscillatory ventilation for adult respiratory distress syndrome: let's get it right this time. Crit Care Med 1997;25:906-8.

3. Amato MB, Barbas CS, Medeiros DM, et al. Effect of a protective-ventilation strategy on mortality in the acute respiratory distress syndrome. $\mathrm{N}$ Engl J Med 1998;338:347-54.

4. Ventilation with lower tidal volumes as compared with traditional tidal volumes for acute lung injury and the acute respiratory distress syndrome. The Acute Respiratory Distress Syndrome Network. N Engl J Med 2000;342:1301-8.

5. Stewart TE, Meade MO, Cook DJ, et al. Evaluation of a ventilation strategy to prevent barotrauma in patients at high risk for acute respiratory distress syndrome. Pressure- and Volume-Limited Ventilation Strategy Group. N Engl J Med 1998;338:355-61.

6. Brochard L, Roudot-Thoraval F, Roupie E, et al. Tidal volume reduction for prevention of ventilator-induced lung injury in acute respiratory distress syndrome. The Multicenter Trial Group on Tidal
Volume Reduction in ARDS. Am J Respir Crit Care Med 1998;158:1831-8.

7. Brower RG, Shanholtz CB, Fessler HE, et al. Prospective, randomized, controlled clinical trial comparing traditional versus reduced tidal volume ventilation in acute respiratory distress syndrome patients. Crit Care Med 1999;27:1492-8.

8. de Durante G, del Turco M, Rustichini L, et al. ARDSNet lower tidal volume ventilatory strategy may generate intrinsic positive end-expiratory pressure in patients with acute respiratory distress syndrome. Am J Respir Crit Care Med 2002;165:1271-4.

9. Eichacker PQ, Gerstenberger EP, Banks SM, Cui X, Natanson C. A metaanalysis of ALI and ARDS trials testing low tidal volumes. Am J Respir Crit Care Med 2002;166:1510-4.

10. Esteban A, Anzueto A, Frutos F, et al. Characteristics and outcomes in adult patients receiving mechanical ventilation: a 28-day international study. JAMA 2002;287:345-55.

11. BT Seeman. Safety Warnings Halt National Study on Lung Sickness. Newhouse News Service, 2002. <www.newhousenews.com/archive/story1b091902.html> (Version current at March 12, 2003)

12. Stewart TE. Controversies around lung protective mechanical ventilation. Am J Respir Crit Care Med 2002;166:1421-2. 


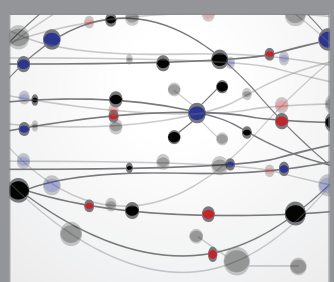

The Scientific World Journal
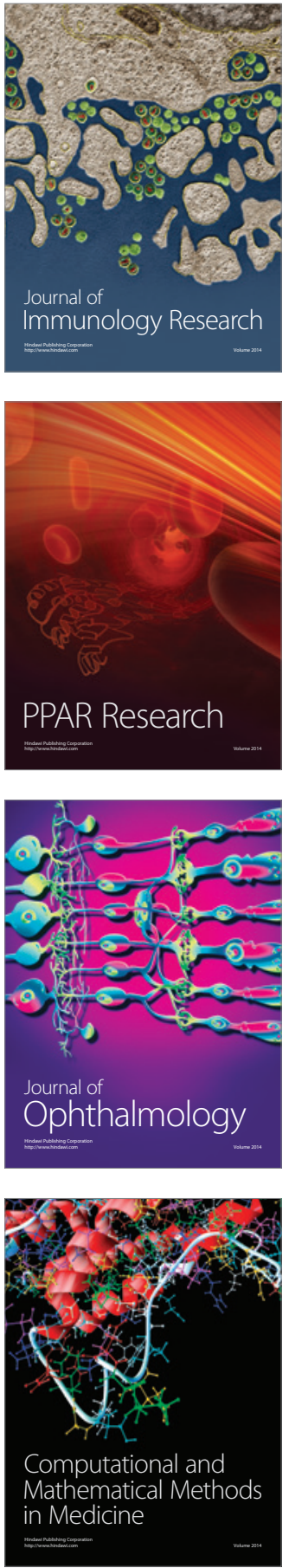

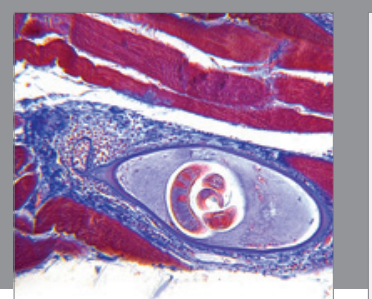

Gastroenterology Research and Practice

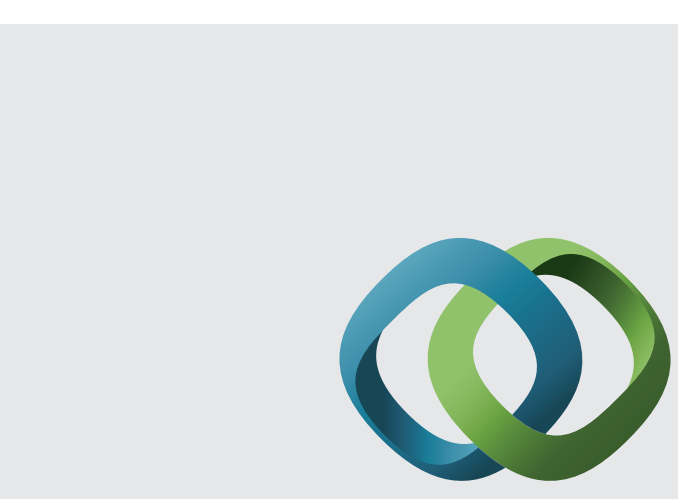

\section{Hindawi}

Submit your manuscripts at

http://www.hindawi.com
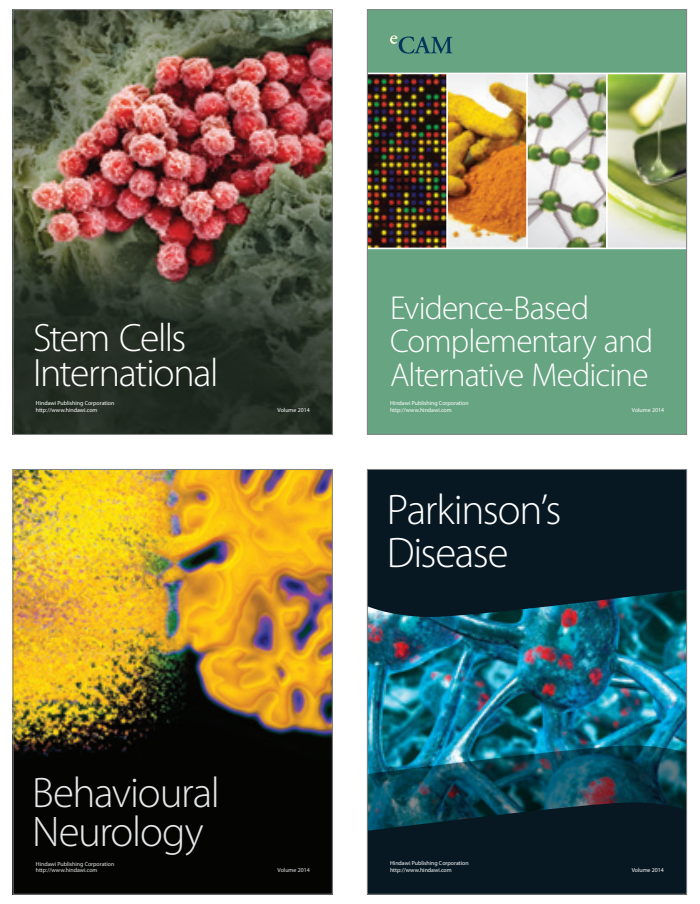
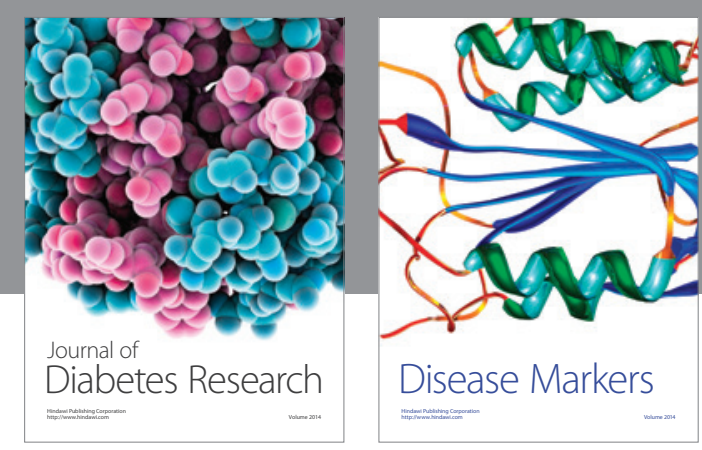

Disease Markers
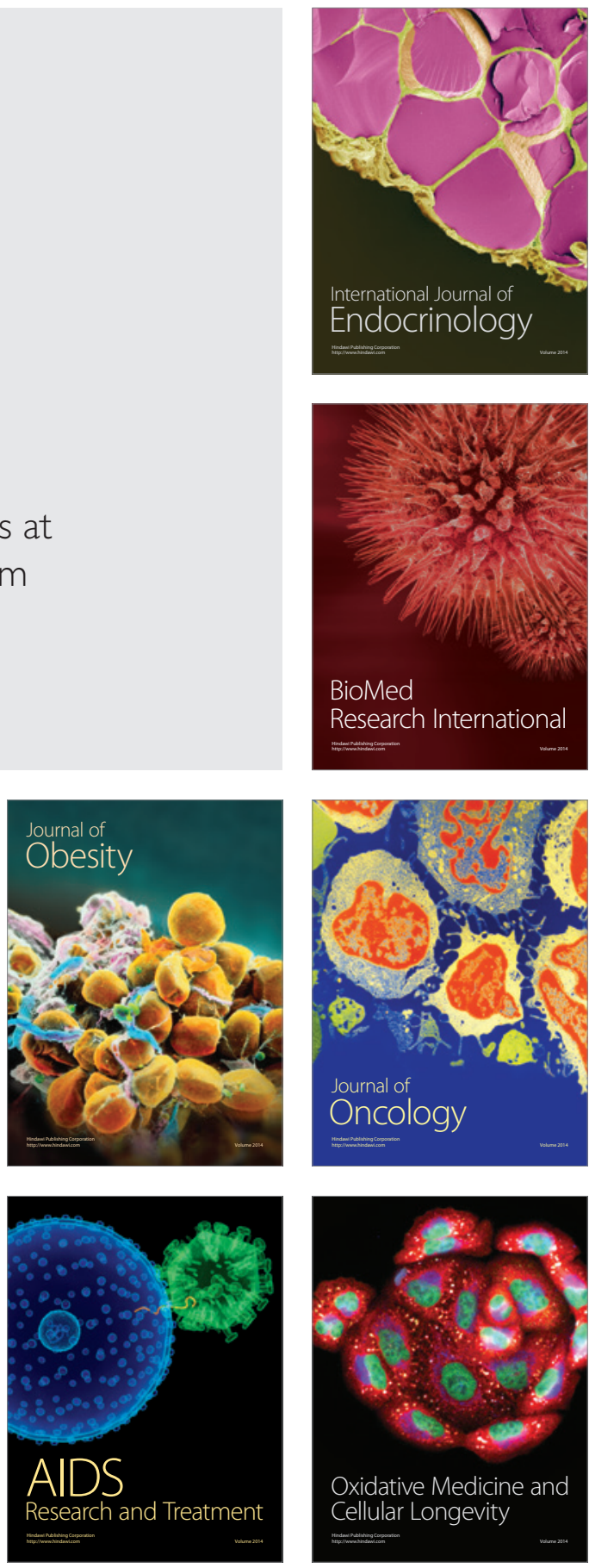